\title{
Exploring the Acetone Evaporation and Airborne Neurotoxicity Bioassay against Adult Mosquito in the Enclosed Environment of Peet Grady Chamber
}

\author{
Jahangir Kamaldin ${ }^{1 *}$ and Zairi Jaal ${ }^{2}$ \\ ${ }^{1}$ Advanced Medical and Dental Institute, Universiti Sains Malaysia, 13200 Kepala Batas, Penang \\ ${ }^{2}$ Vector Control Research Unit, Universiti Sains Malaysia, 11800 Minden, Penang \\ *Corresponding author: jahangirkamaldin@gmail.com
}

A R T I C L E I N F O
Article history:
Received date : 21 June 2018
Revised date: 26 July 2018
Accepted date: 8 August 2018
Available online at:
http://inajac.lipi.go.id
Keywords:
Acetone, Evaporation, Toxicity,
Mosquito, $\quad$ Volatile Organic
Compound.

\begin{abstract}
Mosquito has comprehensive and sensitive olfactory neuro-sensory located at antenna utilized for detecting airborne organic compounds in search of blood host. Mosquito is also known to have a similar neurotransmitter function with human at neural synapses e.g. acetylcholinesterase, esterases and oxidases enzymes. Thus, there is potential use mosquito in predicting neurotoxicity of exogenic volatile organic compounds (VOC) e.g. manufactured acetone. Hence, the study evaluates the suitability to conduct bioassay of VOC neurotoxicity against mosquito in Peet Grady chamber that is commonly used for insecticidal bioassay. Acetone as the representative of VOC is easily evaporized at laboratory temperature of 26 to $29^{\circ} \mathrm{C}$ without heating. The acetone evaporation profile on liquid surface and porous solid surface under the Peet Grady chamber is studied to as certain the consistency of vaporization rate with homogenized distribution. The study showed that the acetone has shown consistent vaporization rate of $23 \mathrm{mg} / \mathrm{min}$ from liquid surface and 116.3 $\mathrm{mg} / \mathrm{min}$ from porous surface (filter paper) without heating, in a linear regression of very high positive correlation $(r=1.000)$ between time and mass of acetone vaporization. However, the non-homogenized distribution of acetone vapours in the Peet Grady chamber directly affected the accuracy to elucidate the neurotoxicity bioassay against mosquitoin term of mosquito knockdown. The study suggests the positioning of mosquitoes in the Peet Grady chamber should be lower than the point of acetone vaporization, whereby the mosquitoes are knockdown by acetone vapours within the 20th minute upon reaching concentration of 170.3 to $196.1 \mathrm{ppm}$.

(C) 2018 Indonesian Journal of Applied Chemistry. This is an open access article under the CC BY-NC-SA license (https://creativecommons.org/licenses/by-nc-sa/4.0/).
\end{abstract}

\section{INTRODUCTION}

\subsection{Acetone}

Acetone has a chemical formula of $\left(\mathrm{CH}_{3}\right)_{2} \mathrm{CO}$ and registered number 67-64-1 under the Chemical Abstracts Service (CAS). It is classified under ketone functional group of chemicals with molar mass of $58.1 \mathrm{gmol}^{-1}$. It is a common laboratory reagent [1]. It is one of the chemical substances under the group of volatile organic compounds (VOC) that are known to exist as liquids at room temperature of 20 to $28{ }^{\circ} \mathrm{C}$. Molar mass of these compounds may vary but their boiling points are relatively lower while their vapor pressures are highercompared to other organic compounds. These make them easy to vaporize from a liquid phase to a gas phase.
Vaporization may occur either by evaporation or boiling but for VOCs their high vapor pressure leads to a fast evaporation, i.e. transition from the liquid phase to gas phase on a surface at a temperature below boiling point [2][3].

In addition to the unique physical and chemical properties of individual compounds, the evaporation rate of VOC is also dependent on the saturation of vapor pressure. In an enclosed atmosphere, saturation will reach when liquids evaporate to gaseous phase equilibrium with gaseous condense back to the liquid phase. Saturation point may differ in relation to space volume and atmospheric temperature. Acetone is one of the common VOC that have a different vapor pressure of 
$181.72 \mathrm{mmHg}$ and $231.06 \mathrm{mmHg}$ at a temperature of $20^{\circ} \mathrm{C}$ and $25^{\circ} \mathrm{C}$, respectively [4]. It is demonstrated that saturated vapor pressure of acetone increased from $5.186 \mathrm{kPa}$ (ca. $38.898 \mathrm{mmHg}$ ) to $15.049 \mathrm{kPa}$ (ca. $112.876 \mathrm{mmHg}$ ) when the temperature is increased from $-10{ }^{\circ} \mathrm{C}$ to $10{ }^{\circ} \mathrm{C}$ [3]. In other studies, it is found that acetone evaporation is largely governed by surface temperature, wind temperature and velocity, and droplet size [5][6][7].

Besides the emission of acetone at a consistent evaporation rate, the concentration of vaporized acetone in the atmosphere may decline due to the adsorption on the surface made of porous or ionic materials. The adsorption process of acetone is reported to be linear at low pressure ranges while adsorption capacity is relatively higher than other VOCs due to its low boiling point and high vapor pressure [8]. There is also the possibility that acetone is adsorbed by indoor airborne particles. VOCs are associated with the particles through the combination of adsorption via surface interactions involving Van De Waals and Lewis acid/base forces, and absorption via insertion into an organic or aqueous layer on a particle's surface [9].

Information on the acetone volatility in the above paragraphs clearly described the importance of standardizing and controlling key experimental conditions, i.e. room, surface and ambient temperature, draught, air volume and material of enclosure to be used in evaluating biological activity of acetone against mosquito. In general, the combination of these conditions has an overall impact on the evaporation rate, consequently affecting the dose of acetone for being vaporized. It is also crucial to understand the profile of the acetone evaporation as to ensure a dose of treatment is adequate and consistent throughout a study. Hence, the main objective of the study is to determine the evaporation rates of acetone from the liquid surface and porous surface in an enclosed air volume.

\subsection{Neurotoxicity of Acetone}

Acetone is mass production for almost two million tons that is used mainly as a solvent and an intermediate in chemical-based production, e.g. papers, plastics, pharmaceuticals and paints industries. It is also known to be used as an extraction solvent for fats and oils, and as a precipitation agent in sugar and starch purification. Besides bulk volume of acetone utilization in manufacturing, its unique character of fast vaporizing liquid solvent made it to be commonly found in consumer products, namely adhesives, cleaners, paints and even nail polish removers. Such wide use of acetone has created unlimited risks of exposure due to emission at occupational and home environments [4] [10] [11].

Although human is exposed to acetone from various anthropogenic sources as described in previous paragraph but it is not foreign to the body system because it is endogenously produced in liver, lung and kidney and all over the body during fatty acid oxidation, whereby enzymatic breakdown of acetoacetate by decarboxylase spontaneously releases acetone with acetyl coenzyme-A as an intermediate metabolite during this process. However, the anthropogenic sources increase body burden to eliminate excessive acetone via mechanisms of urine excretion, air exhalation and mainly by enzymatic metabolism (cytochrome P450 isozymes) that degrades acetone to acetate and formate. Acetone in the form of vapor poses a high probability of exposure during human respiration as it is rapidly absorbed, whereby approximately $50 \%$ of inhaled acetone is absorbed in the respiratory tract (excluding nasal cavity) and distributed by blood circulation within 30 minutes [4].

It is interesting to note that acetyl coenzyme-A, which is instrumental in the process of producing acetone is also an important compound in the production of acetylcholine, a neurotransmitter in the human and dipterans nervous system. Furthermore, acetate that is generated from the degradation of acetone is also generated in the breakdown of acetylcholine by esterase, and it is a raw material for the formation of acetone. The role of acetyl coenzyme-A and acetate in both processes create a question as 
to whether direct absorption of exogenous acetone into the nervous system would create imbalance of acetyl coenzyme-A and acetate concentrations in neurons due to competition in both processes and subsequently interrupting the normal function of neural cholinergic signal transmission activated by acetylcholine [4]. Thus, it is hypothesized that such imbalance could be the reason of acute symptoms i.e. dizziness, headache and loss of consciousness experienced by workers over exposed to acetone [12] [13] [14]. Studies in recent decades also showed that acetone adversely effects on the mammalian nervous system, including humans similar to insecticides [15] [13] [14].

Exposure of insects to high doses of volatile organics is suspected to cause adverse effects, including knockdown and death [16]. Interestingly, acetone is also reported to show neurotoxicity in arthropods [17] and exhibited its insecticidal activity of $100 \%$ mortality against Tribolium confusum, a stored-product pest when fumigated at a concentration of $0.0123 \% \mathrm{v} / \mathrm{v}$ for a duration of 48 hours [18]. A treatment of $123 \mu \mathrm{l}$ of acetone vaporized in 1 liter air is reported to kill Ephestia kuehniella at the adult, larval and egg stages, within three days [18]. Insect including mosquito is known to have similar neurotransmitters function with human at neural synapses e.g. acetylcholinesterase, esterases and oxidases enzymes.

In view of acetone capability to cause adverse effects to human and insect. Therefore, after understanding the evaporation profile of acetone, the second objective of the study is to investigate whether the vaporized acetone in the enclosed environment of the Peet Grady Chamber is capable to cause neurotoxicity signs in mosquito i.e. causing mosquito non-capable to coordinate movement (knockdown) due intoxication, resulting for not being able to stand, walk or normal fly. The Peet Grady Chamber is a standard test arena for the evaluation of biological performance of neurotoxic insecticide against flying insect pests such as mosquitoes and flies [19] [20] [21] [22] [23].
The sign of adverse effect is also linked to dose variation [24] [9] [25] [26], e.g. time interval of mosquito exposure to actual concentration of vaporized acetone. It is a challenging factor to study because acetone's characteristics are colorless and odorless. However, due to high volatility and nonreactive solvent properties, it provided high confidence of quick vaporization to the targeted concentrations of acetone, with an assumption that $100 \%$ vaporization from liquid phase occurs at temperatures above $25^{\circ} \mathrm{C}$ [4]. The quick vaporization time from liquid to gas phase is based on the assumption of acetone's "rapid - equal - random" distribution within an enclosed container, below saturation threshold [3].

\section{MATERIALS AND METHODS}

\subsection{Evaporation of Acetone from Liquid Surface}

The Peet Grady chamber (Figure 1) with inner volume of $5.832 \mathrm{~m}^{3}(1.8 \times 1.8 \times 1.8 \mathrm{~m})$, is selected for the purpose of this study. The chambers are located in a laboratory in the Vector Control Research Unit, School of Biological Sciences, Universiti Sains Malaysia, Penang, Malaysia. The chamber is specially designed and constructed for the testing of biological efficacy of household insecticides products against flying insects [21]. The inner surface of these chambers is made from thick glass and/or Formica ${ }^{\circledR}$, which are known to be hard, durable, resistant to organic insecticide sorption and allows stainless cleaning without residue. Such built characteristics are prioritized to prevent sorption of vaporized acetone on the walls of the chambers. The chambers are also draughttight, equipped with local exhaust ventilation with a velocity above $0.5 \mathrm{~ms}^{-1}$ to remove airborne particles, protected from direct sunlight or direct heat source. The chamber ambient temperature is dependent on the laboratory air-conditioning system because the chambers are not equipped with the independent cooling system. 
Acetone for the experiments is manufactured by Merck KGaA, Germany with product name of "Acetone for analysis EMSURETM ACS, ISO, Reagent Ph Eur." The product CAS is 67-64-1 with purity > $99.7 \%$ [27]. In reference to the safety data sheet published by Merck KGaA Germany [28], the laboratory ambient temperature is maintained at 26 to $29{ }^{\circ} \mathrm{C}$ to facilitate vaporization while minimizing the flammability risk of acetone. The laboratory did not have a specific system to regulate air humidity; throughout the experiment, the relative humidity varied from 60 to $90 \%$. Therefore, the chamber is manually wiped and air-dried to prevent a substantial change of relative humidity.

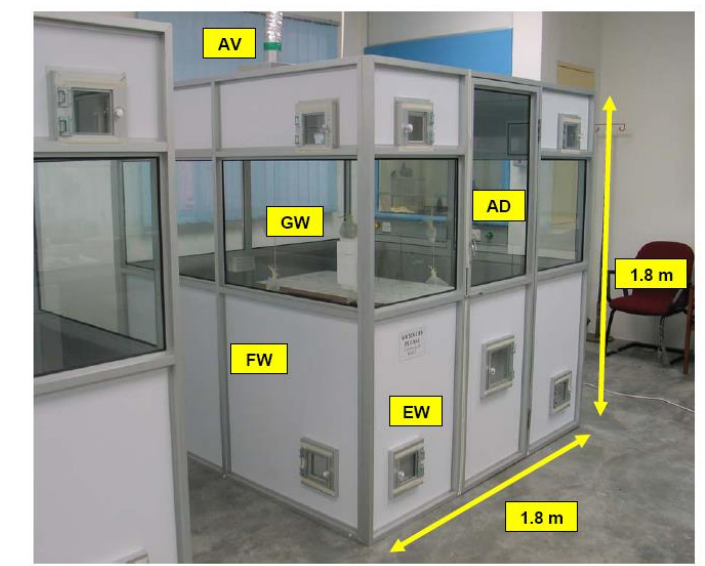

\section{Legend}

FW: Walls made from Formica ${ }^{\circledR}$ - aluminium provide structural support

GW: Walls made from glass - aluminium serve as observation window

$A D$ : Door for entry to and exit from the chamber

EW: Small square windows serve as access ports for the experimental purpose

$A V$ : Exhaust ducting equipped with active ventilator to replace or refresh the chamber's air.

Note: Dimension in meters

Fig. 1. Structural built of Peet Grady chamber

A top pan weighing balance (Dragon 3002, Mettler-Toledo) with a maximum mass capacity of $3100 \mathrm{~g}$ and smallest readable mass of $0.01 \mathrm{~g}$ is placed approximately in the centre of each chamber. The water bubble is readjusted to the centre to level the balance and weighing accuracy is verified using verified standard weights. It is ensured that weight measurement display of the balance is viewable from the window of the chamber.
The windows and doors of the chambers are closed after a period of 30 minutes to ensure ambient atmospheric temperature in the chambers. Through a window of the chamber, a volume of $50 \mathrm{ml}$ acetone is poured into a $100 \mathrm{ml}$ glass beaker and then is placed on the balance, and the window is closed. The mass of the acetone is recorded at every minute for a period of 30 minutes. After 30 minutes, the beaker containing acetone is removed from the balance and closed with a lid to prevent further evaporation. Subsequently, the chamber's air is ventilated using local exhaust ventilator with a velocity of above $0.5 \mathrm{~ms}^{-1}$ for a period of 30 minutes. The experiment is replicated five times.

\subsection{Evaporation of Acetone from Porous Surface}

Filter paper (Whatman® ${ }^{\circledR}$ No. 1, diameter $15 \mathrm{~cm}$ ) is used as the porous surface matrix. The maximum number of filter papers that could be placed on the chamber's floor is 73 pieces due to the limitation of floor space and working space. The volume of required acetone had to be adjusted due to the physical feasibility in performing the experiments and safety concerns. Liquid holding capacity of a piece of filter paper is pre-determined by dripping acetone using a $10 \mathrm{ml}$ hypodermic syringe on the filter paper that is placed on a flat surface. The liquid holding capacity is quantified based on a maximum volume of acetone absorbed by the filter paper until the whole surface of the paper is wet. The maximum liquid holding capacity of each five filter papers is treated in the range from 5.6 to $6.0 \mathrm{ml}$. However, $3 \mathrm{ml}$ is selected as the optimum volume of acetone for treatment on each filter paper, in order to reduce the time taken to treat the filter papers and prevent overflowing of acetone to neighbouring filter papers.

A walking path is designated between the filter paper rows to facilitate application of acetone. The doors and windows of the chamber are closed from inside and a volume of $3 \mathrm{ml}$ acetone is applied to every filter papers by using pre-filled $50 \mathrm{ml}$ hypodermic syringes. The total volume of acetone applied 
is $219 \mathrm{ml}$. Upon completion of acetone application, the time taken for the whole wet filter papers to dry (wet mark disappeared) is recorded. Upon complete disappearance of the wet mark from all the filter papers, the chambers are ventilated for at least 60 minutes before opening the chamber for disposal of the filter papers. The chambers are also manually wiped and air-dried for approximately one hour without heating. This experiment is replicated five times using fresh filter papers and cleaned chambers. The laboratory temperature and relative humidity are maintained at 25 to $27^{\circ} \mathrm{C}$ and 60 to $90 \%$, respectively. The chambers are protected from direct sunlight.

\subsection{Neurotoxicity of Airborne Acetone against Adult Mosquito}

Mosquitoes for the experiments, namely Aedes aegypti were sourced from an established laboratory colony maintained at 25 to $29{ }^{\circ} \mathrm{C}, 50$ to $90 \%$ relative humidity and a photoperiod of 12:12 hours [29] [30] in the insectarium of the Vector Control Research Unit, School of Biological Sciences, Universiti Sains Malaysia, Penang, Malaysia. In general, mosquitoes were bred using the general rearing technique [31] with several improvements in larval diet [32], adult diet [33] and feeding techniques [34]. Male and female mosquitoes were fed with sugar solution only [33] and caged together to allow mating. Female mosquitoes were regularly provided with mouse blood for eggs production [35] [31]. A bowl lined with a moist filter paper wrapped in a cone shape is placed in the holding cage for eggs collection up to several days. Female Aedes mosquitoes tend to deposit eggs on the moist filter paper. The deposited eggs on the filter paper were immersed into a pan of chlorine-free water or distilled water to hatch the eggs.

Newly hatched Aedes larvae were transferred into polyethylene containers, which contain chlorine-free water or distilled water. The larvae were fed with commercial fish pellets on daily basis depending on the larvae consumption. The water in the container is refilled regularly after 3-5 days or whenever necessary, depending on the water turbidity. When the larvae in the container start to molt into $3^{\text {rd }}$ instar larvae or pupae, the container is transferred into a holding cage and given sufficient time for the adults to emerge. After the adult mosquitoes completely emerged, the polyethylene container is removed from the holding cage. The newly emerged adults were sugar-fed by placing a cotton wick soaked in a glass vial filled with sugar solution. It is ensured that only sugar-fed females were collected for experiments in this chapter [36] [37]. In addition, considering the possibility of genomic drift between generations, care is taken to ascertain that the mosquitoes utilized for the experiments were from a single generation, i.e. F50.

The results of the acetone evaporation rate are utilized in designing the experiment to study the neurotoxicity of airborne acetone against adults mosquitoes without direct surface contact. The inner surface of the chamber is wiped several times with a damp cloth using a mild soap solution and tap water for 30 minutes. Then the inner surface is fandried and actively ventilated using an electrical exhaust system for at least for 15 minutes. Subsequently, a cylindrical cage containing several mosquitoes is hanged inside in the centre of the chamber for a period of 30 minutes to verify the chamber is free from chemical contaminants. Once verified clean, the cylindrical cage is removed and small pocket nets (approximate 7 x 5 x 1 $\mathrm{cm}$ ) each containing a fresh female Aedes aegypti mosquito is hanged at every corner of the chamber at a height of approximately 50 $\mathrm{cm}, 80 \mathrm{~cm}$ and $110 \mathrm{~cm}$ from the chamber's floor. A modified bench $(120 \mathrm{~cm}$ length $\mathrm{x} 80$ $\mathrm{cm}$ width $\times 80 \mathrm{~cm}$ height) is placed at the center of the chambers and covered with 56 pieces of filter papers (Whatman No. 1, diameter $=15 \mathrm{~cm}$ ) as illustrated in Figure 2 . The use of filter papers is based on the favourable results of the experiments described in section 2.2. All the chamber's windows and door were closed and sealed with tapes. Approximately $220 \pm 10 \mathrm{ml}$ acetone is equally dispensed over the 56 
pieces of filter paper, with average $4 \mathrm{ml}$ acetone per filter paper. The maximum concentration of acetone upon complete homogenized evaporation within the Peet Grady would be $29.6 \mathrm{ppm}$ based on the approximated density of acetone $0.785 \mathrm{~g} / \mathrm{ml}$ at $25^{\circ} \mathrm{C}$.

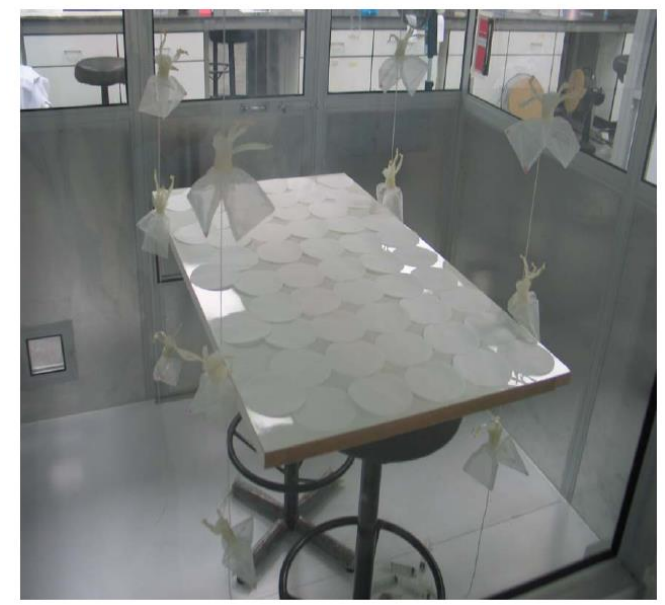

Note: Acetone is dispensed on filter papers placed on the bench at height of $80 \mathrm{~cm}$.

Fig. 2. Spatial positioning of pocket nets at different heights of $50 \mathrm{~cm}, 80 \mathrm{~cm}$ and $110 \mathrm{~cm}$ from the floor of the test chamber and at a distance of $50 \mathrm{~cm}$ from the wall of the chamber.

After complete dispensing the acetone, mosquitoes in the pocket nets were observed for knockdown up to 60 minutes. In consideration that the treated acetone on filter papers is not completely evaporated, the actual airborne acetone is measured using a VOC meter i.e. a real-time handheld portable photoionization detector (MultiRAE IR Gas Monitor) with an attached air-sampling tube. The tube's inlet is $80 \mathrm{~cm}$ from the chamber's floor. The VOC meter is calibrated using a standard calibration kit containing isobutylene gas for measurement of VOC within 0 to 2000 ppm detection limits with 1 ppm resolution. The total VOC concentration in the chamber's atmosphere is recorded at every interval of 5 minutes to estimate the amount of acetone that has been vaporized in the chamber. After the 60th minute observation, the chamber is left for 24 hours to allow complete vaporization and degradation over time. The residue of acetone remaining in the chamber is verified using the VOC meter. The experiment replication is limited to 5 times in view the experimental procedure had high risks of fire due to acetone high flammability and auto-ignition.

\section{RESULT AND DISCUSSION}

\subsection{Comparison of Evaporation Rate of Acetone between Liquid and Porous Surfaces}

The mean cumulative liquid mass reduction of acetone from the $100 \mathrm{ml}$ glass beaker shown by Figure 3 at each minute observed up to 30 minutes under the Peet Grady chamber experimental conditions plotted a linear positive regression with a very high positive correlation $(\mathrm{r}=1.000, \mathrm{P}<$ $0.001)$ between the vaporized acetone mass and time. The linear regression line fit allowed the modelling of linear algorithms for high accuracy prediction of acetone evaporation at $\mathrm{y}=0.023 \mathrm{x}$. The " $\mathrm{y}$ " in the equation represents vaporized mass while " $x$ " denotes the time, which results in evaporation rate of $0.023 \mathrm{~g} / \mathrm{min}$, approximately 0.029 $\mathrm{ml} / \mathrm{min}$ based on the estimated density of $0.785 \mathrm{~g} / \mathrm{ml}$ at $25^{\circ} \mathrm{C}$.

While the use of filter paper as a matrix for the treatment of acetone has magnified significantly $(\mathrm{P}<0.01)$ the evaporation rate to $14.81 \mathrm{ml} / \mathrm{min}(11.63 \mathrm{~g} / \mathrm{min})$, facilitated by feasibility to treat a larger volume of acetone i.e. $219 \mathrm{ml}$ on the proportionate surface area of the 73 pieces of filter papers.

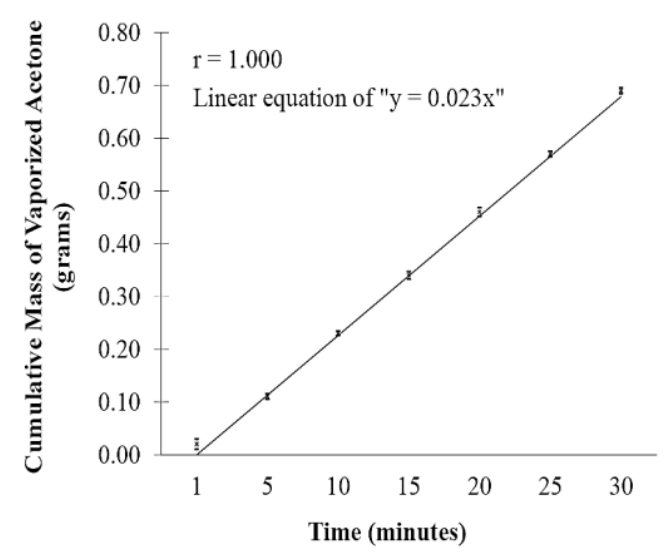

Fig. 3. The profile of acetone evaporation from the $100 \mathrm{ml}$ glass beaker at floor level under the Peet Grady chamber experimental conditions. 
Post experiment disposal of the used filter paper found it to be not completely dry after the conduct of the experiment. Indicating the disappearance of the wet mark during the 30 minutes observation, may not represent total evaporation of the treated acetone on the filter papers. It is suspected caused by lacks of air circulation in the chamber via active ventilation [38] [22] [39]. However, it is not feasible to introduce such ventilation because it would not allow accumulation of airborne acetone at a designated concentration as required for the experiments. The idea of introducing a motorized-fan within the chambers to circulate the air is also not feasible because of the potential fire hazard posed by the flammable acetone.

Nevertheless, the disappearance of the wet mark after the $219 \mathrm{ml}$ acetone treatment from all the filter papers without any formation of condensation droplets on the chambers glass walls, suggest that the evaporation of acetone using this technique is capable to increase treatment dose up to 171.92 gram $(29.5 \mathrm{~g} / \mathrm{m} 3$, equivalent to $29.5 \mathrm{ppm}$ ) within an evaporation time of 14.8 minutes. The improved evaporation rate is likely been contributed by the larger surface area and acetone volume temporary holding by the filter papers. The results of the acetone evaporation profile in the Peet Grady chamber provided essential information towards designing a neurotoxicity study of vaporized (airborne) acetone against the adult mosquitoes as the insect model for screening presence of neurotoxin in the air.

\subsection{Neurotoxicity of Airborne Acetone against Adult Mosquito}

The knockdown time of mosquitoes confined in the pocket nets at the respective spatial positioning is exhibited in Table 1. It is noticed that only mosquitoes that were placed at the height $80 \mathrm{~cm}$ and $50 \mathrm{~cm}$ from the acetone vaporizing point on the bench have been knockdown within a duration of 18.95 to 22.17 minutes. Regardless, whether the mosquitoes are placed at corner A, B, C or $\mathrm{D}$, with insignificant difference $(\mathrm{P}>0.05)$ using ANOVA. It is also evident that all mosquitoes positioned at $110 \mathrm{~cm}$ that is above the acetone vaporizing point were not affected throughout the 60 minutes exposure time with a significant difference $(\mathrm{P}<0.05)$.

Acetone has shown consistent vaporization rate of $23 \mathrm{mg} / \mathrm{min}$ from liquid surface and $116.3 \mathrm{mg} / \mathrm{min}$ from the porous surface (filter paper) as described in section 3.1.

Table 1. Mean \pm standard deviation of knockdown time of female Aedes aegypti mosquitoes at different spatial dimension upon exposure to $220 \mathrm{ml}$ acetone vaporized from filter paper in a Peet Grady Chamber (1.8 x $1.8 \times 1.8 \mathrm{~m}$ ).

\begin{tabular}{|c|c|c|c|}
\hline $\begin{array}{l}\text { Corner of the } \\
\text { chamber }\end{array}$ & $\begin{array}{l}\text { Target dose of } \\
\text { acetone }\left(\mathrm{ml} / \mathbf{m}^{3}\right)\end{array}$ & $\begin{array}{l}\text { Height from chamber's floor } \\
\text { y axis }(\mathrm{cm})\end{array}$ & $\begin{array}{l}\text { Mean } \pm \text { Standard Deviation } \\
\text { Knockdown Time* (minute) }\end{array}$ \\
\hline $\mathrm{A}$ & 31 & $\begin{array}{c}50 \\
80 \\
110\end{array}$ & $\begin{array}{c}20.23 \pm 4.32 \mathrm{a} \\
19.35 \pm 3.88 \mathrm{a} \\
\text { No Knockdown }\end{array}$ \\
\hline $\mathrm{B}$ & 31 & $\begin{array}{c}50 \\
80 \\
110\end{array}$ & $\begin{array}{c}20.17 \pm 5.48 \mathrm{a} \\
20.88 \pm 2.47 \mathrm{a} \\
\text { No Knockdown }\end{array}$ \\
\hline $\mathrm{C}$ & 31 & $\begin{array}{c}50 \\
80 \\
110\end{array}$ & $\begin{array}{c}21.03 \pm 2.21 \mathrm{a} \\
20.87 \pm 4.33 \mathrm{a} \\
\text { No Knockdown }\end{array}$ \\
\hline $\mathrm{D}$ & 31 & $\begin{array}{c}50 \\
80 \\
110\end{array}$ & $\begin{array}{c}22.09 \pm 2.37 \mathrm{a} \\
18.95 \pm 5.93 \mathrm{a} \\
\text { No Knockdown }\end{array}$ \\
\hline
\end{tabular}

Note:

* Mean values that are followed by different alphabets in a column were found to be significantly different based on Student-Newman-Keuls Mean Comparisons Test $(\alpha=0.05)$. 
Thus, based on the acetone's consistent vaporization, it is expected that the vaporization of acetone in the Peet Grady chamber will deliver a gradual increase of dose and reach the maximum targeted dose of $30 \mathrm{ppm}$ in the chamber within 15 minutes. Eventually, mosquitoes placed at any $x-y$ spatial position $(50-50 \mathrm{~cm}, 50-80 \mathrm{~cm}$ and 50 $110 \mathrm{~cm}$ ) within the chamber would be equally exposed to the vaporized acetone.Logically, the mosquitoes placed at the position of 50-80 $\mathrm{cm}$, which is closest to the source of acetone vaporization is anticipated to be knockdown first compared to mosquitoes positioned at $50-50 \mathrm{~cm}$ and $50-110 \mathrm{~cm}$, whereby both positions were $30 \mathrm{~cm}$ vertically away from the source of acetone evaporation. However, the results observed were surprising because the mosquitoes positioned at $30 \mathrm{~cm}$ above the source of acetone evaporation, i.e. at position 50-110 cm were not knockdown. While mosquitoes placed at positions $50-50 \mathrm{~cm}$ and $50-80 \mathrm{~cm}$ were consistently knockdown at the similar time interval in all five replicates.

The measurement of VOC (Table 2) shows that acetone evaporation inside the Peet Grady chamber took a duration of 25 minutes to reach a saturation level of $258 \mathrm{ppm}$ when measured in proximity of the vaporization point i.e. at the bench level $(80 \mathrm{~cm}$ from the floor), which is almost 9 folds higher than the originally estimated dose of $30 \mathrm{ppm}$. Even though acetone is highly volatile but there is the possibility that the vaporized acetone did not disperse in all direction randomly as anticipated for a gas phase molecule to behave. There is a possibility that the confined atmosphere of the chamber creates a vapour liquid-gas saturation point that limits the dispersion of acetone in gas phase. Moreover, the acetone molecules are heavier than the air, its higher molar mass $(58 \mathrm{~g} / \mathrm{mol})$ compared to other major gas molecules in the chamber's atmosphere, e.g. nitrogen (14 $\mathrm{g} / \mathrm{mol})$ and oxygen $(16 \mathrm{~g} / \mathrm{mol})$ contributing $78 \%$ and $21 \%$ of the chamber's air, respectively. Hence, the vaporized acetone molecules were subjected to greater gravitational force downwards upon losing its kinetic energy after vaporizing in an upwardmotion.

This situation could further be facilitated by the higher gaseous pressure of nitrogen and oxygen that is more than $100 \mathrm{kPa}$ compared to acetone at $25 \mathrm{kPa}$, making the top portion of the chamber less available for vaporized acetone. Consequently, the heavier acetone vapours accumulated at the bottom of the chamber, at a height of $80 \mathrm{~cm}$ and lower. Thus, explains the faster mosquito knockdown time shown by mosquitoes at the $50-80 \mathrm{~cm}$ and $50-50 \mathrm{~cm}$ positions $[2][4][6][7]$.

Table 2. Mean \pm standard deviation of total VOC measurement upon the evaporation of $220 \mathrm{ml}$ acetone from filter paper at the height of $80 \mathrm{~cm}$ in a Peet Grady chamber $(1.8 \times 1.8 \times 1.8 \mathrm{~m})$.

\begin{tabular}{|c|c|c|c|}
\hline $\begin{array}{c}\text { Time } \\
\text { (minute) }\end{array}$ & $\begin{array}{l}\text { Target dose of } \\
\text { airborne acetone } \\
(\mathrm{ppm})\end{array}$ & $\begin{array}{l}\text { Point of measurement height from } \\
\text { chamber's floor - y axis }(\mathrm{cm})\end{array}$ & $\begin{array}{l}\text { Mean } \pm \text { standard deviation of } \\
\text { measured total VOC* }(\mathrm{ppm})\end{array}$ \\
\hline 0 & 0 & 80 & $21.8 \pm 8.4$ \\
\hline 5 & 10 & 80 & $38.0 \pm 17.5$ \\
\hline 10 & 20 & 80 & $87.3 \pm 28.6$ \\
\hline 15 & 30 & 80 & $128.0 \pm 21.0$ \\
\hline 20 & 30 & 80 & $183.2 \pm 12.9$ \\
\hline 25 & 30 & 80 & $222.0 \pm 33.4$ \\
\hline 30 & 30 & 80 & $246.8 \pm 37.3$ \\
\hline 35 & 30 & 80 & $257.6 \pm 39.6$ \\
\hline 40 & 30 & 80 & $258.4 \pm 40.6$ \\
\hline 45 & 30 & 80 & $258.8 \pm 40.3$ \\
\hline 50 & 30 & 80 & $256.8 \pm 37.9$ \\
\hline 55 & 30 & 80 & $247.8 \pm 40.2$ \\
\hline 60 & 30 & 80 & $240.6 \pm 38.3$ \\
\hline $\begin{array}{c}1440 \\
\text { (24 hours) }\end{array}$ & 0 & 80 & $15.9 \pm 9.5$ \\
\hline
\end{tabular}


The occurrence of accumulation is reflected by the results of the total VOC measurement shown at Table 2. The targeted concentration of $30 \mathrm{ppm}$ vaporized acetone is achieved within the first 10 minutes of the experiment. But the total concentration of VOC kept rising up to $258 \mathrm{ppm}$ with markable increase during the first 25 minutes. The results also supports the postulation that the acetone vapour accumulated at the lower zone of the chamber, i.e. below the evaporation point and is not equally dispersed. Although theactual dispersion of acetone vapour is not as anticipated but it is evident that mosquitoes are knockdown by acetone vapourson the $20^{\text {th }}$ minute timeline upon reaching concentration of 170.3 to 196.1 ppm in the large Peet Grady chamber.

\section{CONCLUSION}

Overall, the findings of the experiments strongly suggest that spatial dimension affects the vaporization patterns of acetone from the source of evaporation. Subsequently causing the non-homogenized distribution of acetone vapours in an enclosed large environment such as Peet Grady chamber with volume above $5 \mathrm{~m}^{3}$. Hence, the evaporation pattern will directly affect the accuracy of the neurotoxicity bioassay against mosquito or other flying insects conducted under the Peet Grady chamber conditions. Nevertheless, the neurotoxicity effects in term of mosquito knockdown can be elucidated using the Peet Grady chamber with the positioning of mosquitoes should be lower the point of acetone vaporization.

\section{ACKNOWLEDGMENT}

The authors wish to thank Vector Control Research Unit, School of Biological Sciences, Universiti Sains Malaysia for providing the mosquito supplies and the use of the household insecticide bioassay laboratory. Heartiest appreciation to all the students and staff involved in the conduct of the study. The research is performed under the funding provided by the Ministry of Science,
Technology and Innovation (MOSTI) thru Grant IRPA 06-02-05-00031 EAR.

\section{REFERENCES}

[1] M.H. Gabb and W.L. Latchem. A Handbook of Laboratory Solution. Andre Deutsch Limited, London, UK. 1967.

[2] R.T. Morrison and R.N. Boyd. Organic chemistry, 6th edition. Prentice Hall, New Jersey, US. 1992.

[3] C.C. Marco, M. Ilham, C. Dominique, J. Jacques and V. Andree. (2004). "Saturated vapour pressure of aroma compounds at various temperatures. "Food Chemistry, 85, pp 221 - 229. 2004.

[4] IPCS. Environmental Health Criteria 207:Acetone. International Programme on Chemical Safety (IPCS), World Health Organization, Geneva. 1998.

[5] D. Bohland G. Jackson. "Experimental study of the spill and vaporization of a volatile liquid." Journal of Hazardous Materials, 140, pp. 117 - 128. 2007.

[6] W.P. Jones, S. Lyra and A.J. Marquis. "Large Eddy Simulation of evaporating kerosene and acetone sprays." International Journal of Heat and Mass Transfer, 53, pp. 2491 - 2505. 2010.

[7] R.S. Khadayate, J.V. Sali, and P.P. Patil. "Acetone vapour sensing properties of screen printed WO3 thick films." Talanta, 72, pp. 1077 - 1081. 2007.

[8] Q. Fang, Z. Lizhong and Y. Kun. "Adsorption behaviours of volatile organic compounds (VOCs) on porous clay heterostructures (PCH)." Journal of Hazardous Materials, 170, pp. 7 12. 2009.

[9] J.W. Charles. "Indoor/outdoor connections exemplified by processes that depend on an organic compound's saturation vapor pressure." Atmospheric 
Environment, 37, pp. 5455 - 5465 . 2003.

[10] W.N. William and J.W. Charles. "Cleaning products and air fresheners: exposure to primary and secondary air pollutants." Atmospheric Environment, 38, pp. 2841 - 2865. 2004.

[11] S. Tung-Sheng, W. Kuen-Yuh, C. Hong-I, C. Cheng-Ping, C. Ho-Yuan, H. Yi-Shiao and L. Saou-Hsing. "The development and regulation of occupational exposure limits in Taiwan." Regulatory Toxicology and Pharmacology, 46, pp. 142 - 148. 2006.

[12] E. Kiesswetter, M. Blaszekewicz, R.R. Vangala and A. Seeber. "Acute exposure to acetone in a factory and ratings of well-being. "Neurotoxicology", 15, pp. $597-602$. 1994.

[13] G. Wang, G. Maranelli, L. Perbellini, E. Raineri, and F. Brugnone. "Blood acetone concentration in normal people and in exposed workers $16 \mathrm{~h}$ after the end of the workshift."International Archive of Occupational and Environmental Health, 65, pp. 285 289. 1994.

[14] T. Satoh, K. Omae, H. Nakashima, T. Takebayashi, H. Matsumura, T. Kawat, M. Nakaza, and H. Sakurai. "Relationship between acetone exposure concentration and health effects in acetate fiber plant workers." International Archive of Occupational and Environmental Health, 68,pp. 147 153. 1996.

[15] D.G. Ross. "Acute acetone intoxication involving eight male workers." American Occupational Hygiene Journal, 16, pp. 73 - 75. 1973.

[16] A.S. Miguel and V. Eugenio. "Enzymes involved in the detoxification of organophosphorus, carbamate and pyrethroid insecticides through hydrolysis. "Toxicology Letters, 128, pp. $215-228.2002$.
[17] J.L. Ruben, F. Lucrecia, and H.V. Julio. "Effects of Lindane and Acetone on the Development of Larvae of the Southern King Crab (Lithodes antarcticus, Jaquinot)." Bulletin of Environmental Contamination and Toxicology, 46, pp. 185 - 192. 1991.

[18] I. Tunc, F. Erler and O. Calis. "Insecticidal activity of acetone vapors. Journal of Stored Product Research,33, pp. 181 - 185. 1997.

[19] J.R. Busvine. A Critical Review of the Techniques for Testing Insecticides. London School of Hygiene and Tropical Medicine. London, UK. 1970.

[20] SIRIM. Specification for mosquito coils, Part 2: method for the evaluation of biological efficacy - glass chamber method (first revision). Malaysian Standard, MS 23: Part 2: 1996, ICS: 65.100, SIRIM, Shah Alam, Malaysia. 1996.

[21] SIRIM. Specification for mosquito coils, Part 3: method for the evaluation of biological efficacy - Peet Grady chamber method (first revision). Malaysian Standard, MS 23: Part 3: 1998, ICS: 65.100, SIRIM, Shah Alam, Malaysia. 1998.

[22] H. Wolfgang, J. Oliver and W. Olaf. "Suitability of small environmental chambers to test the emission of biocides from treated materials into the air. "Atmospheric Environment, 37, pp. 5477 - 5483. 2003.

[23] Lee, S.C. and Wang, B. "Characteristics of emissions of air pollutants from mosquito coils and candles burning in a large environment chamber. "Atmospheric Environment, 40, pp. 2128 - 2138. 2006.

[24] M. Christian. "Exposure assessment of air pollutants: a review on spatial heterogeneity and indoor/outdoor/ personal exposure to suspended particulate matter, nitrogen dioxide and ozone. "Atmospheric Environment, 35, pp. 1 - 32. 2001. 
[25] H. Fromme, T. Lahrz, M. Piloty, H. Gebhardf, A. Oddoy and H. Rüden. "Polycyclic aromatic hydrocarbons inside and outside of apartments in an urban area". Science of the Total Environment, 326, pp. 143 - 149. 2004.

[26] B. Brian, D. Ishai and Y. Bruno. Contaminant Geochemistry: Interactions and Transport in the Subsurface Environment. SpringerVerlag Berlin Heidelberg, Germany. (2008).

[27] MERCK. Acetone for analysis EMSURE® ACS, ISO, Reag. Ph Eur. Merck KGaA, Frankfurter Str. 250, 64293 Darmstadt, Germany. 2010.

[28] MERCK. Safety data sheet: acetone. Merck KGaA, Darmstadt, Germany. 2006.

[29] K. Jahangir, H.H Yap, and C.R. Adanan. "Response of female Aedes aegypti (L.) to colored baits under illumination of monochromatic light. "Journal of Bioscience, 8, pp. 133-140. 1997.

[30] K. Jahangir, J. Zairi, and C.Y. Lee. "Biting rhythms of Aedes mosquitoes under confined area". The $4^{\text {th }}$ Kelantan Health Conference, 6-7 $7^{\text {th }}$ July 2005 in Kota Bharu, Malaysia. 2005.

[31] N.L. Chong, H.H. Yap, Chong, A.S.C., Adanan, C.R. and Lim, S.Y. "Laboratory culture of selected household insect pests." In: Workshop Manual on Biology and Control of Urban Pests, VCRU Science Series No. 7 (Yap, H.H., Chong, N.L. and Lee, C.Y., eds), p. 87-90. Penang: Vector Control Research Unit, Universiti Sains Malaysia. 1998.

[32] K. Jahangir, J. Juskasmini and M.M. Saira Banu. "Fish pellets as feed for larvae of Aedes aegypti. The $42^{\text {nd }}$ Annual Scientific Seminar of Malaysian Society of Parasitology and Tropical Medicine, 1-2 March 2006, Ipoh, Perak, Malaysia. 2006.
[33] K. Jahangir, J. Juskasmini, M.B. Noorhusna, and O. Nor Asima. "Fortified drinks for mosquito? "The $42^{\text {nd }}$ Annual Scientific Seminar of Malaysian Society of Parasitology and Tropical Medicine, 1-2 March 2006, Ipoh, Perak, Malaysia. 2006

[34] K. Jahangir, H.H. Yap, J. Zairi, C.Y. Lee. "The effect of cloth wetted with sugar solution and water on prolonging the lifespan of Aedes aegypti (Linnaeus) and Aedes albopictus (Skuse) under laboratory condition". Journal of Tropical Biomedicine, 20, pp. 145-152. 2003.

[35] J.C. Jones and B.V. Madhukar. "Effects of sucrose on blood avidity in mosquitoes". Journal of Insect Physiology,22, pp. 357 - 360. 1976.

[36] K. Jahangir, J. Zairi, C.Y. Lee and M.M. Saira Banu. "Feeding on sugar solution does not inhibit attraction of female Aedes to human". The $1^{\text {st }}$ ASEAN Congress of Parasitology and Tropical Medicine, 23-25 March 2004 in Kuala Lumpur, Malaysia. 2004.

[37] K. Jahangir, C.Y. Lee and J. Zairi. "The Effects of Sugar and Animal Blood Availability on the Attraction of Aedes (Stegomyia) spp. to human". The International Conference of Urban Pests, $13-16^{\text {th }}$ July 2008. Budapest, Hungary. 2008.

[38] C. Nicola. "A new detailed chemical model for indoor air pollution". Atmospheric Environment,41, pp. 11641179. 2007.

[39] L.Z. Zhangand J.L. Niu. "Modeling VOCs emissions in a room with a single-zone multi-component multilayer technique". Building and Environment, 39, pp. 523 - 531. 2004. 